\title{
Theme: Pediatric Nephrology
}

丹

Utility of different blood pressure measurement components in childhood to predict adult carotid intima-media thickness (Hypertension. 2019;73:335-41)

Childhood blood pressure (BP) levels predict adult subclinical atherosclerosis. It is well known, among adults, that elevated carotid artery intima-media thicknessis associated with cardiovascular disease and stroke. The best childhood BP component for prediction has not been determined. This study comprised 5925 children aged 3 to 18 years who were followed into adulthood (mean (SD) follow-up 25.8 (6.2) years). Childhood BP was measured by using a standard mercury sphygmomanometer. Carotid intimamedia thickness $\geq 90$ th percentile was used to define subclinical atherosclerosis. In age- and sex-adjusted analyses, area under the receiver operating characteristic curves for systolic BP (SBP) showed significantly improved prediction compared with diastolic $\mathrm{BP}$ or mean arterial pressure. Combining different BP components did not improve prediction over SBP measurement alone. Based on the associations with adult carotid intima-media thickness, cut points for elevated SBP were $105 \mathrm{mmHg}$ for 3- to 6-year-old boys, $108 \mathrm{mmHg}$ for 3- to 6-year-old girls, $108 \mathrm{mmHg}$ for 7- to 12-yearold boys, $106 \mathrm{~mm} \mathrm{Hg}$ for 7- to 12-year-old girls, $123 \mathrm{~mm} \mathrm{Hg}$ for 13- to 18 -year-old boys, and $115 \mathrm{~mm} \mathrm{Hg}$ for 13 - to 18 -year-old girls.

Mycophenolate mofetil versus levamisole in frequently relapsing nephrotic syndrome (Kidney Int. 2019;95:210-8)

This single-center, randomized, open-label trial from India enrolled 149 children ages 6-18 years with frequently relapsing or steroiddependent nephrotic syndrome, to either receive therapy with MMF (750-1000 mg/m² daily) or levamisole $(2-2.5 \mathrm{mg} / \mathrm{kg}$ on alternate days) for 1 year; prednisolone was discontinued by 2-3 months. The frequency of relapses was similar in MMF and levamisole treatment groups. Relapse rates declined to almost onethird of baseline for both treatment groups. Therapy with MMF was not superior to levamisole in terms of the proportions of participants with sustained remission $(40.8 \%$ vs. $34.2 \%)$, frequent relapses $(14.5 \% v s .16 .4 \%)$, or treatment failure.

Optimizing AKI definitions during first postnatal week (Pediatr Res. 2019;85:329-38)

AWAKEN (Assessment of Worldwide Acute Kidney injury Epidemiology in Neonates) is the largest neonatal Acute kidney injury (AKI) study to date. It is the first multi-center, multi-national project and the first to include infants hospitalized in the neonatal intensive care unit across the gestation age spectrum. All neonates admitted to 24 participating neonatal intensive care units from four countries (Australia, Canada, India, United States) between January 1 and March 31, 2014, were screened. Of 4273 neonates screened, $2022(47.3 \%)$ met study criteria. Neonates with $\geq 1$ serum creatinine ( $\mathrm{SCr}$ ) on postnatal days 1-8 were assessed. They compared the mortality predictability of $\mathrm{SCr}$ absolute $(\geq 0.3$ $\mathrm{mg} / \mathrm{dL}$ ) vs percent ( $\geq 50 \%$ ) rise. They also determined the optimal absolute, percent, and maximum SCr thresholds that provide the highest mortality area under curve (AUC) and specificity for different GA groups. The $\geq 0.3 \mathrm{mg} / \mathrm{dL}$ rise outperformed $\geq 50 \% \mathrm{SCr}$ rise. The optimal SCr thresholds to predict AUC and specificity were $\geq 0.3$ and $\geq 0.6 \mathrm{mg} / \mathrm{dL}$ for $\leq 29$ weeks GA, and $\geq 0.1$ and $\geq 0.3 \mathrm{mg} / \mathrm{dL}$ for $>29$ week GA. The maximum $\mathrm{SCr}$ value provides great specificity.

Three-monthly bolus vitamin D supplements (1000 vs $400 \mathrm{IU} /$ day) for prevention of bone loss in children with difficult-to-treat nephrotic syndrome (Paediatr Int Child Health. 2018;38:251-60).

There are no uniform consensus guidelines regarding the optimum dose of calcium and vitamin D dosage in children for osteoprotection. This parallel-group, open-label, randomized clinical trial was done to compare the efficacy of three-monthly bolus vitamin D supplementation (1000 vs $400 \mathrm{IU} /$ day) to prevent bone loss in children with difficult-to-treat nephrotic syndrome (DTNS). Sixty children aged 1-18 years with DTNS [37 with frequently relapsing NS (FRNS), 13 steroid-dependent NS (SDNS) and 10 steroid-resistant NS (SRNS)] were enrolled and block randomized. In Group A, oral vitamin D was administered at a bolus dose of 90,000 IU every three months (calculated for a period of three months at 1000 IU/day). In Group B, vitamin D (cholecalciferol) was administered as a bolus dose of 36,000 IU every three months (calculated for a period of three months at $400 \mathrm{IU} /$ day). The proportionate change in bone mineral content (BMC) was studied by dual energy $X$-ray absorptiometry (DEXA) scan (baseline $v s$. after 12 months). The proportionate change in BMC was not significantly different between the two groups (median proportionate change in BMC in Group A $13.4 \%$ vs $11.6 \%$ in Group $\mathrm{B}, P=0.22)$. Overall, BMC increased in both groups ( $96.7 \%$ in each). None of the patients had hypercalciuria at the end of the study. Authors concluded that three-monthly bolus vitamin D dosing regimens administered either as 1000 or $400 \mathrm{IU} /$ day prevent bone loss in children with DTNS who require long-term steroids. Further studies are required to demonstrate the superiority of the former regimen over the latter.

SidHaRTh KUMAR SETHI sidsdoc@gmail.com
Indian Pediatrics commemorates World Kidney day-March 14, 2019. The theme for this year is "Kidney Health for Everyone Everywhere." See details at www.worldkidneyday.org

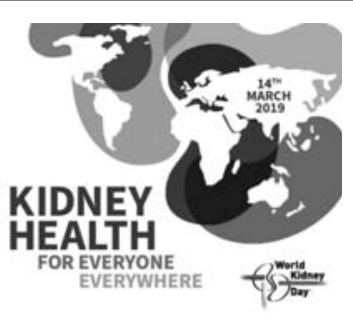

VOLUME 56-MARCH 15, 2019 